\title{
EFFECTIVENESS OF AN INTERSECTION VIOLATION WARNING SYSTEM
}

\author{
Dawn Marshall, Robert Wallace, James Torner, \& Michelle Birt-Leeds \\ University of Iowa \\ Iowa City, Iowa, USA \\ Email: dawn-marshall@uiowa.edu
}

\begin{abstract}
Summary: People age 65 years and older are the fastest growing segment of the U.S. population and the fastest growing sector of the driving population. When compared to other age groups, older drivers are overrepresented in intersection crashes (Subramanian \& Lombardo, 2007; Braitman et al., 2006), and approximately half of the charges in fatal intersection crashes are for failure to obey the traffic control device. This project explored an in-vehicle warning system for failure-to-obey (running a stop sign or stop light) violations. Participants who were not using the system made nearly three times as many didnot-stop errors (27\%) than participants who were using the system (10\%). This effect was most pronounced in older drivers with more risk factors associated with crashes; however, the effect of age group was not statistically significant.
\end{abstract}

\section{INTRODUCTION}

In 2005, 36 million people in the United States, or 12 percent of the population, were aged 65 years and older. The Census Bureau estimates that about 13 percent of the population will be over 65 by 2010 and that the percentage will increase to 16.4 percent by 2020 as the "baby boomers" enter this age group (He et al., 2005). As individuals move into the older population, most continue to drive. When compared to the entire U.S. driving population, older drivers are not dramatically overrepresented in terms of driver fatalities in terms of percentage of drivers. Older drivers accounted for $14 \%$ of driver fatalities in 2007 and $15 \%$ of the licensed drivers in 2006 (NHTSA, 2007). However, older drivers travel approximately half the number of miles of those under age 65 (Lyman et al., 2002). As a result, the crash rate per mile driven is about twice as great for older drivers. Some of these fatalities can be attributed to the increased fragility of older drivers. In other words, older drivers are more likely to be killed than younger drivers involved in similar crashes. Fragility does not explain the entire picture of older driver risk, however. With age, many drivers experience declines in vision, hearing, reaction times, and cognitive and motor abilities (Staplin et al., 1998). Even conscientious drivers must accommodate for these physical and mental challenges.

Age alone does not explain the higher incidence of accidents among older drivers, so factors that co-exist with greater age must be considered. Persons with cognitive or physical impairment from specific conditions, such as Alzheimer's disease or Parkinson's disease, may be at increased risk of motor vehicle crashes (Gorrie et al., 2007; Johansson et al., 1997; Dobbs et al., 1998; Rizzo et al., 1997; Wood et al., 2005; Zesiewicz et al., 2002; Uc et al., 2006; Uc et al., 2007). Motor functions and physiological factors such as loss of mobility in the head and neck may challenge drivers when entering an intersection (Isler et al., 1997). Vision problems are a relevant specific risk for crashes. Older drivers with low vision reported more problems with both near and distance acuity and with physical obstructions than did older drivers with normal 
vision (McGregor \& Chaparro, 2005), and decreased visual acuity and contrast sensitivity were associated with self-reported difficulty in high-risk driving situations (McGwin et al., 2000). Other physical indicators, such as at least one fall in the past year or foot reaction time, may be risk factors for crashes in older women (Margolis et al., 2002). Failure-to-obey and failure-toyield situations are often considered under the single topic of negotiating intersections. However, there is a clear division in the successful negotiation of intersections: a safe and appropriate stop (failure to obey), then proceeding through the intersection (failure to yield). This work focused on failure-to-obey situations and an in-vehicle system designed to warn drivers when it predicted a violation.

\section{METHOD}

The experimental design used 36 participants from three age-related groups; 'middle-normal'(25$55)$, 'older normal'(>65) and 'older at-risk'(>65) drivers. The participants were presented two levels of vehicle system presence (present and not present). The protocol included a screening for general health and driving criteria and a process to classify potential participants as "normal" or "at risk" based on their scores relating to cognitive impairment and health and mobility factors that are related to crash risk in older drivers. Participants completed one 15-minute drive in the NADS-1 driving simulator on an urban and arterial four-lane road network with a posted speed limit of $35 \mathrm{mph}$. During the simulator drive, they passed through several controlled intersections. An intersection violation warning system, which was present for half the participants, was designed to provide alerts when a driver was likely to violate a red light or stop sign at an intersection. The system used vehicle location, traffic signal state, and timing to determine probability of violation. The system alert included three display components: a visual icon, an auditory alert, and a brake pulse. Following the simulator drives, participants completed short surveys about their experience in the simulator.

\section{Test Devices}

Driving Simulator. The NADS-1 driving simulator, owned by NHTSA and located at The University of Iowa, comprises a 13-degree-of-freedom motion base with a 24-foot-diameter dome in which a Chevrolet Malibu cab was mounted for this study. Inside the dome, the cab was mounted to the floor through four hydraulic actuators. The dome can rotate about its vertical axis by 330 degrees in each direction and was mounted on top of a traditional hydraulic hexapod, which in turn was mounted on two belt-driven beams that could move independently along the $\mathrm{X}$ and $\mathrm{Y}$ axes in a 64-foot-by-64-foot bay. The visual system consisted of eight liquid crystal display (LCD) projectors that project a 360-degree photo-realistic virtual environment. The front three projectors had a resolution of $1600 \times 1200$. The right and left projectors had a resolution of 1280 x 1024. The three projectors in the back had a resolution of 1024 x 768 . All scenery was updated and displayed 60 times per second. A complete statement of capabilities can be found in the NADS Statement of Capabilities (National Advanced Driving Simulator, 2007).

Warning system. The in-vehicle system used vehicle location, traffic signal state, and timing to determine probability of violation. The system was active throughout the simulator drives in which it was present, with an assumed communication range of 300 meters prior to each intersection. A violation was predicted by a time to arrival to the stop bar of an intersection of 
less than $t_{c r i t}$ given the following equation derived from the CICAS-V critical stop distance equation (Maile et al., 2008):

$$
t_{\text {crit }=t_{\text {react }}} \frac{V_{i}}{2\left(a_{\text {lim }}\right)}
$$

where $t_{\text {react }}$ (reaction time) was $1.5 \mathrm{~s}$, and $a_{\text {lim }}\left(v_{i}\right)$ (assumed rate of deceleration for a given velocity) was specified as a constant $0.35 \mathrm{~g}$. No violation warning was given if the participant's time to arrival to the stop bar was less than the time to red (Maile et al., 2008). In other words, if the participant could make it through the yellow light before the light turned red, no violation warning was given. In addition, no violation warning was given if the participant's speed was below $5 \mathrm{mph}$. The visual alert was reset after a warning 5 seconds after the participant had come to a stop (speed $<0.4 \mathrm{mph}$ ), or when the participant had crossed the midpoint in the intersection. The system alert included three display components: a visual icon, an auditory alert, and a brake pulse. The visual icon display was mounted on the center of the dashboard, is shown in

Figure 1.

At a pre-established time to arrival at an intersection the icon became blue and steady and on warning activation, the icon became red and flashed at $4 \mathrm{~Hz}$ with a $50 \%$ duty cycle (125 ms on, $125 \mathrm{~ms}$ off) until 5 seconds after the participant had crossed the stop line. The auditory alert was presented simultaneously with the visual icon and was a voice alert. Two sound files were utilized: "stop light" or "stop sign" for systempredicted violations at traffic-signal-controlled intersections and stop-sign-controlled intersections, respectively. The alerts were recorded using a female voice and were presented at approximately $75 \mathrm{db}$ measured from the driver's head. The brake pulse followed the same profile as the examples provided in the CICAS-V work (Perez et al., 2008); however, adjustments were made to accommodate the motion washout in NADS-1. The brake pulse was triggered immediately before the onset of the visual and auditory warnings, such that deceleration would reach $0.10 \mathrm{~g}$ at the same time as the visual and auditory warnings with a total pulse duration of approximately 0.6 seconds and the peak pulse between 0.25 and 0.35 seconds after the onset

Figure 2. of the visual/auditory warning,
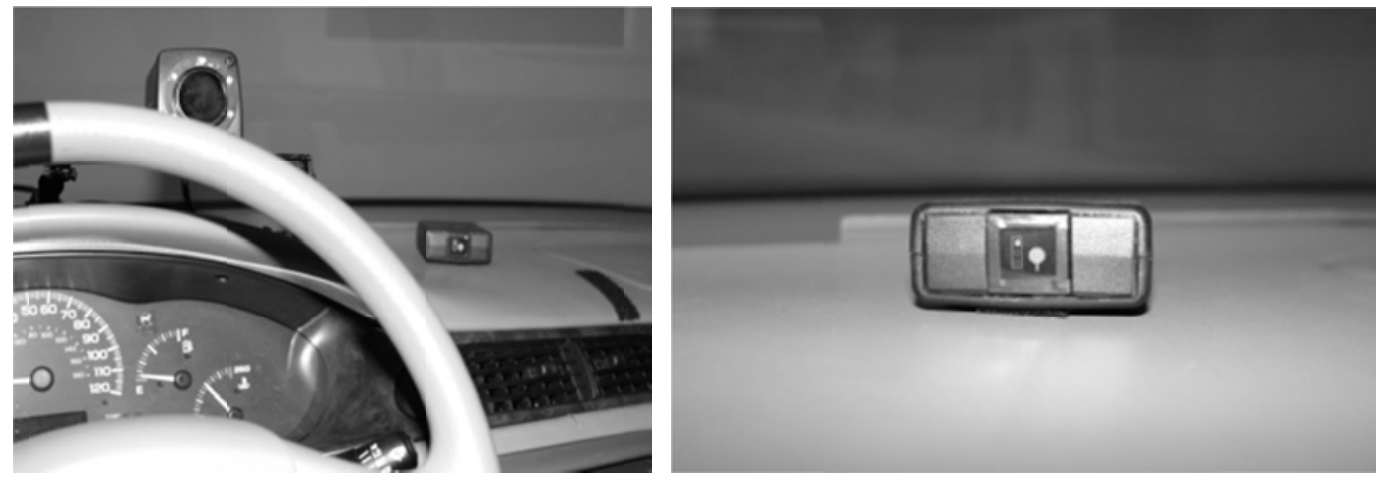

Figure 1. Visual alert icon 


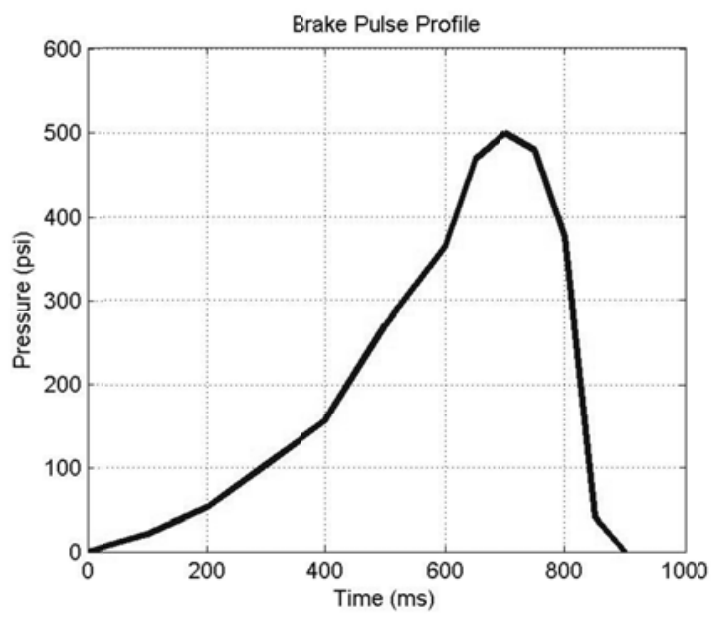

Figure 2. Brake pulse profile

Driving Scenarios. The road network consisted of a main four-lane road with a posted speed limit of $35 \mathrm{mph}$. The environment for the simulator drive included two urban sections, each with six intersections, separated by an arterial section of shallow curves with two intersections. Experimental events occurred at four intersections in the second urban section, as well at two stop signs with crosswalks placed in the arterial section. Events included a stop sign visually obstructed by a tree, three unobstructed stop signs, a red light, and a light that changed from green to yellow as a heavy truck lead vehicle approached the intersection then from yellow to green while the truck obscured the driver's view of the traffic light in the right lane, however the yellow light above the left lane was still visible.

\section{Protocol}

Participants. Participants fit into one of three groups: "normal” middle drivers (25-55 years old), “normal” older drivers (>65 years old), and “at risk” older drivers (>65 years old). “At risk” was defined as participants whose scores on a Health and Mobility Classification questionnaire or the Telephone Interview for Cognitive Status (TICS) showed they had physical and/or cognitive risk factors for involvement in a driving accident. "Normal” was defined as participants whose scores did not reach the thresholds on the Health and Mobility questionnaire and the TICS that would put them in the "at risk" category. Thirty-six, twelve from each age group participated in the study.

Study visit. Participants completed the informed consent document, a driving history and demographic survey. They then completed several evaluations for risk factors associated with traffic accidents in older drivers. After the paperwork and evaluations were completed, the participant went through a self-paced PowerPoint presentation that described the in-vehicle system and what they could expect in the simulator. Following the PowerPoint presentation, participants completed the 15-minute study drive. During the first part of the drive, participants became acquainted with the simulator cab and practiced stopping in the simulator. After the drive, participants were asked to complete a post-drive questionnaire consisting of questions about the warning system. There were two versions, one for participants who experienced the invehicle system and one for those who did not. 


\section{RESULTS}

There was a clear overall benefit associated with the presence of the warning system. There were nearly three times more did-not-stop outcomes without the system (27\%), than when the system was present (10\%); see Figure 3. This was particularly true in situations where the presence of stop sign or the state of a traffic light would be more difficult for drivers to detect.

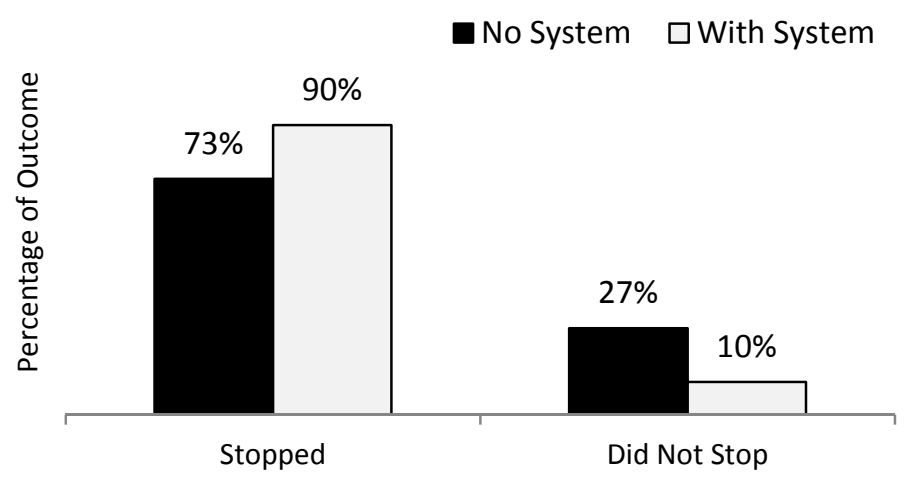

Figure 3. Frequency of outcome by system presence

It is possible that drivers most at risk of crashes may benefit most from the presence of the system as implied by the greatest change in did-not stop outcomes in the older at-risk group, see Figure 4, even though the trend did not reach statistical significance.

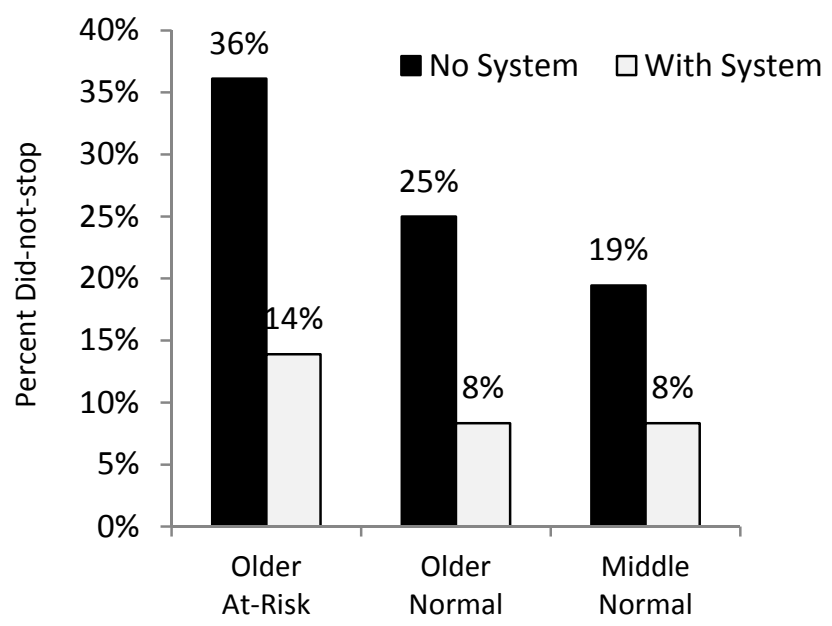

Figure 4. Frequency of outcome by age-risk group

\section{DISCUSSION}

The benefit associated with the system was also seen in the stopping position data. Participants who experienced the system warning stopped instead of driving through the intersection, resulting in more stops past the stop bar, but before the collision zone. There was also a general perception among those who experienced the system that the system improved driving safety and that it aided drivers in driving more carefully. Whereas, those who did not experience the system 
tended to disagree that the system would make driving safer and disagreed it would help them drive more carefully than they normally would. The disagreement with these statements by those who did not experience the system may indicate that experiencing the system reveals its benefit to users. The positive perception of the system coupled with the benefit seen in did-not-stop outcomes indicates that an intersection violation warning system would be welcomed and used by drivers.

\section{Limitations}

It should also be noted that this experimental design and protocol included simple situations at intersections and did not examine potential unintended consequences of the presence of the system. The data from this study are from a first-time single use of the system. How drivers would respond to the system over time is unknown and over reliance is a possibility. It is also not clear from this work how drivers would respond to the warning system in more complex situations such as intersections with cross traffic present, the presence of tailgating vehicles, and the presence of pedestrians crossing the road at intersections. Additionally, only one system specification was used and systems using different alert timings and combinations may not show the same benefits. This study showed a system benefit; however there are a number of untested conditions (traffic situations, systems differences, levels of system experience) which could produce differing levels of safety impact.

\section{ACKNOWLEDGMENTS}

This research was funded under National Highway Traffic Safety Administration (NHTSA) contract DTNH22-06-D-00043 Task Order No. 3.

\section{REFERENCES}

Braitman et al. (2006). Crash and Error Types of Older Drivers' Intersection Crashes. IIHS.

Dobbs, A., Heller, R., \& Schopflocher, D. (1998). A comparative approach to identify unsafe older drivers. Accident Analysis and Prevention , 30 (3), 363-370.

Gorrie, C., Rodriguez, M., Sachdev, P., Duflou, J., \& Waite, P. (2007). Mild neuritic changes are increased in the brains of fatally injured older motor vehicle drivers. Accident Analysis and Prevention , 39 (6), 1114-1120.

He et al. (2005). Sixty-Five Plus in the United States: 2005. Special Population Studies. US Census Bureau. P23-209.

Isler, R., Parsonson, B., \& Hansson, G. (1997). Age related effects of restricted head movements on the useful field of view of drivers. Accident Analysis and Prevention , 29 (6), 793-801.

Johansson, K., Bogdanovic, N., Kalimo, H., Winblad, B., \& Viitanen, M. (1997). Alzheimer's disease and apolipoprotein E epsilon 4 allele in older drivers who died in automobile accidents. Lancet , 349 (9059), 1143-1144.

Lyman S. Ferguson S., Braver E. \& Williams A. (2002). Older Driver involvements in police reported crashes and fatal crashes: Trends and projections. Injury Prevention. 8:116-120. 
Maile, M., Ahmed-Zaid, F., Caminiti, L., Lundberg, J., Mudalige, P., \& Pall, C. (2008). Cooperative Intersection Collision Avoidance System Limited to Stop Sign and Traffic Signal Violations (CICAS-V), System Design Specification-Infrastructure. USDOT: Washington, DC.

Margolis, K., Kerani, R., McGovern, P., Songer, T., Cauley, J., \& Ensrud, K. (2002). Risk Factors for Motor Vehicle Crashes in Older Women. The Journals of Gerontology. Series A, Biological Sciences and Medical Sciences , 57 (3), M186-191.

Marottoli, R., Richardson, E., Stowe, M., Miller, E., Brass, L., Cooney, L. J., et al. (1998). Development of a test battery to identify older drivers at risk for self-reported adverse driving events. Journal of the American Geriatrics Society , 46 (5), 562-568.

McGregor, L., \& Chaparro, A. (2005). Visual difficulties reported by low-vision and nonimpaired older adult drivers. Human Factors , 47 (3), 469-478.

McGwin, G. J., Chapman, V., \& Owsley, C. (2000). Visual risk factors for driving difficulty among older drivers. Accident Analysis and Prevention , 32 (6), 735-744.

National Advanced Driving Simulator. (2007, September). NADS statement of capabilities: Submitted in response to NHTSA's request in the Indefinite Quantity Contract (DTNH22-06D-00043) (NADS document no. N07-019). Iowa City, IA: NADS Staff.

National Highway Traffic Administration (2007). Traffic Safety Facts. 2007 Data. DOT HS 810 992

Rizzo, M., Reinach, S., McGehee, D., \& Dawson, J. (1997). Simulated car crashes and crash predictors in drivers with Alzheimer Disease. Archives of Neurology , 54 (5), 545-551.

Staplin L., Lococo K.H, McKnight J. \& Odenheimer G.L. (1998). Intersection Negotiation Problems of Older Drivers, Volume II: Background Synthesis on Age and Intersection driving Difficulties. National Highway Traffic Safety Administration. DOT HS 808850.

Subramanian R., \& Lombardo L. (2007). Analysis of Fatal Motor Vehicle Crashes and Fatalities at Intersection, 1997 to 2004. National Highway Traffic Safety Administration. DOT HS 810 682.

Uc, E., Rizzo, M., Anderson, S., Sparks, J., Rodnitzky, R., \& Dawson, J. (2006). Impaired visual search in drivers with Parkinson's disease. Annals of Neurology , 60 (4), 407-413.

Uc, E., Rizzo, M., Anderson, S., Sparks, J., Rodnitzky, R., \& Dawson, J. (2007). Impaired navigation in drivers with Parkinson's disease. Brain , 130 (9), 2433-2440.

Wood, J., Anstey, K., Kerr, G., Lacherez, P., \& Lord, S. (2008). A multidomain approach for predicting older driver safety under in-traffic road conditions. Journal of the American Geriatrics Society , 56 (6), 986-993.

Wood, J., Worringham, C., Kerr, G., Mallon, K., \& Silburn, P. (2005). Quantitative assessment of driving performance in Parkinson's disease. Journal of Neurology, Neurosurgery, and Psychiatry , 76 (2), 176-180.

Zesiewicz, T., Cimino, C., Malek, A., Gardner, N., Leaverton, P., Dunne, P., et al. (2002). Driving safety in Parkinson's disease. Neurology , 59 (11), 1787-1788. 